\title{
Drug Development for Cystic Fibrosis
}

\author{
Don Sanders ${ }^{1}$ and James Chmiel ${ }^{1}$ \\ ${ }^{1}$ Riley Hospital for Children at Indiana University Health
}

July 1, 2020

\begin{abstract}
The first drug specific for cystic fibrosis (CF) was approved in 1993, and since then several other drugs have been approved. Median predicted survival in people with CF has improved from approximately 30 years to 44.4 years over that same period. In 2020, highly-effective modulators of the cystic fibrosis transmembrane conductance regulator became available to approximately $90 \%$ of people with CF ages 12 years and older. These transformative therapies will surely reduce morbidity and further extend longevity. The drug development pipeline is filled with therapies that address most aspects of CF disease. As survival and $\mathrm{CF}$ therapies have advanced, and the complexity of CF care increases, the process of drug development has become more sophisticated. In addition, detecting meaningful changes in clinical outcomes has become more difficult as the health status of people with CF improves. Design of clinical trials in CF has become more complex, and innovative approaches are required to continue to advance drug development. This review provides a general overview of drug development from the pre-clinical phase through Phase IV. Special considerations with respect to CF are integrated into the discussion of each phase of drug development. The dawn of a new era has arrived for people with CF. As CF care evolves, drug development must continue to evolve as well, until a one-time cure is available to all people with $\mathrm{CF}$.
\end{abstract}

\section{Introduction}

When considering the success and cost of taking a molecule from the laboratory to an approved drug, the statistics are sobering. Of 1,000 candidate therapeutics being considered during the pre-clinical phase, only one will go on to clinical trials(1). Of the candidate drugs that enter testing in humans, only $10 \%$ will go on to be approved by the FDA $(2)$. The average cost of developing one drug approved by the FDA is $\$ 2.6 \mathrm{~B}$ with another $\$ 300 \mathrm{M}$ in post-marketing costs(3). Not only is drug development expensive, it is also time consuming. It typically takes 12-15 years for a drug to go from preclinical testing to approval(4). These statistics account for the pharmaceutical industry mantra, "An early kill is a good kill". The sooner an industry sponsor can eliminate a candidate drug from its portfolio that will ultimately fail, the smaller the financial loss. It is within this backdrop that patients, healthcare providers, and biomedical researchers must operate. These realities often make developing drugs for orphan diseases like cystic fibrosis (CF) difficult. Large pharmaceutical sponsors often focus on diseases that have been shown to respond to treatment or that affect millions of people, where risk of failure is lower and the potential return-on-investment is much greater. Smaller biotechnology firms are often willing to take a chance on orphan diseases where the competition is less but frequently lack the capital to carry drug development to its completion. These smaller firms become dependent on outside support, such as angel funding and venture philanthropy to continue to move drug development forward. Despite these realities, the vision of the CF Foundation, particularly in its development of the Therapeutics Development Network in 1998(5), has led to an astounding number of new therapies being approved for people with CF considering the status of $\mathrm{CF}$ as an orphan disease. More industry sponsors are willing to consider developing new drugs for CF because the financial support of the CF Foundation has mitigated some of the risks associated with drug development and helped smaller firms bridge the gap between angel funding and venture capitalism.

In 1993, the FDA approved dornase alfa, the first drug developed specifically for $\mathrm{CF}(6)$. Since that time, 
multiple drugs have been approved to treat a variety of aspects of CF, including the abnormal cystic fibrosis transmembrane conductance regulator (CFTR) protein, mucus obstruction, bacterial infection, and gastrointestinal and nutritional disease (Figure 1). Many other candidate drugs are currently in clinical trials or preclinical evaluation, including potential therapies that can repair/replace abnormal RNA or DNA (https://www.cff.org/Trials/Pipeline. Accessed June 20, 2020). The development of modulator therapy to address the primary defect in the CFTR protein began in the late 1990s when high-throughput screening was first used to identify small molecules that could potentiate or correct CFTR dysfunction. This culminated with the 2019 approval of elexacaftor/tezacaftor/ivacaftor which provides highly-effective modulator therapy for approximately $90 \%$ of people with CF 12 years of age and older(7). Drug development for CF has become increasingly complex over the last 20 years, and clinical trial endpoints are changing because traditional endpoints are becoming less common events. In this manuscript, we will provide an overview of how a molecule goes from a drug candidate in the laboratory to an approved drug therapy for a patient (Figure 2).

\section{Pre-Clinical Drug Development}

All drug development starts in the laboratory in the phase that is termed pre-clinical development. This process generally takes up to 6 years or longer. When considering drug development, researchers must consider several fundamental questions during the pre-clinical phase. These questions are especially crucial in an orphan disease like $\mathrm{CF}$ where the margin for error is much smaller than for more common diseases. First and foremost, investigators must determine whether there is an unmet need. Are there any therapies available that addresses a certain area of the disease? Are the available therapies insufficient either from an efficacy or safety standpoint? Of the currently approved drugs, are there limitations on availability to everyone, whether due to cost, lack of effectiveness or adverse reactions? Other questions that must be considered include: what aspect of the disease will be targeted? Will the drug have to be active in several organs or just one organ? Within an organ, what process will be targeted? For example, in the lungs, will the drug impact the basic defect, abnormal airway environment, mucus obstruction, chronic infection or exaggerated inflammation (Figure 1)? Is the target a cell, a receptor, or a molecule? Will the drug correct an abnormal protein structure like a CFTR corrector, decrease something produced in excess like a pro-inflammatory mediator, or increase something that is not produced or produced in relatively low concentrations like anti-inflammatory molecules or CFTR in people with two nonsense mutations?

In a complex target like airway inflammation where there are many triggers impacting dozens of molecular pathways that result in the increased production of pro-inflammatory molecules or decreased production of counter-regulatory molecules, it is often difficult to determine the most efficacious target that is also a safe target. These complexities have made developing new anti-inflammatory therapies for CF extremely difficult. Over the last 30 years, dozens of anti-inflammatory drugs being considered for CF have failed along the way during the various phases of drug development(8-10). One candidate drug was actually associated with increased pulmonary exacerbations, the opposite of the intended effect(11). The clinical trial of this investigational product provided a lesson that highlights the importance of having adequate pre-clinical safety data in a disease-relevant animal model(12). The past provides a cautionary tale for all future drug development, not just for anti-inflammatory drugs.

During the pre-clinical phase of drug development, the efficacy of an investigational product is typically demonstrated in both in vitro and in vivo models. During pre-clinical experiments, having the correct assay is the cornerstone. The ideal assay is simple, high-throughput, directly relevant to the intended therapeutic target, and has the ability to discriminate between more or less efficacious analogous molecules. The investigator must consider what outputs should be used to measure efficacy and safety and how those outputs might be adapted for use in a clinical trial. Toxicology studies in disease-relevant animal models are important in order to determine dose-limiting toxicities and establish initial dosages that are likely to be safe in human studies. These are required as a part of the regulatory process and are ultimately geared toward minimizing risk of untoward effects happening in human trials. For example, when studying anti-inflammatory drugs, an important determination is at what dose might an anti-inflammatory (the 
target effect) become an immunosuppressive (an untargeted and potentially deleterious effect)(13)? This points to the importance of studying anti-inflammatory drugs in a representative model system, e.g., a CF mouse model with an active infection similar to what might occur in the human CF airway, versus a dissimilar animal model, e.g., a sterile mouse model. Adequate safety must be established in animals before proceeding to clinical trials in humans. Pre-clinical safety studies should be conducted under good laboratory practice (GLP) guidelines, which is defined as "a quality system concerned with the organisational process and the conditions under which non-clinical health and environmental safety studies are planned, performed, monitored, recorded, archived and reported "(14).

The ultimate goal of the pre-clinical phase of drug development is to file an Investigational New Drug (IND) application with the FDA. The FDA's primary objective in evaluating pre-IND research is to ensure the rights and safety of human subjects are being protected. During the pre-clinical phase, several molecules in the same drug class may be under investigation, but eventually, a single lead compound, typically with a similar, backup compound, must be selected. Prior to the selection of the lead molecule, various modifications are considered to optimize activity and chemical properties to help ensure that the sponsor has the ability to make enough of the investigational product under good manufacturing practice (GMP) guidelines to complete all of the non-clinical safety tests. Under these guidelines, the production of an investigational compound must be consistently of high quality between batches. In the pre-clinical phase, animal studies provide information regarding the pharmacology and toxicology of the molecule under consideration. Animal studies also provide a basis for conducting a clinical trial. Typically, animal studies consist of a rodent species and a large non-rodent species. Once short-term toxicology data are available, a clinical trial can start, but human studies cannot be longer in duration than the duration of animal toxicology studies. For therapies intended for chronic use, six-month animal toxicology data are necessary for registration of the drug. The purpose of the animal studies is to provide information on a dose range or therapeutic window, initial dosages, an estimation of the risk:benefit ratio for humans, identification of target organ toxicities and safety margin, and identification of surrogate markers to monitor during clinical trials(15). At this point, an IND application is submitted to the FDA prior to proceeding to a clinical trial (Box 1). Once the FDA approves an IND, the investigator is able to proceed to human trials.

No overview of the pre-clinical phase of drug development would be complete without some discussion of funding. It is during the pre-clinical phase that collaborations between academic and industry colleagues are often forged. Prior to this point, academicians often secure research funding for pre-clinical studies from government agencies such as the NIH or private foundations such as the Cystic Fibrosis Foundation, amongst other sources. However, for development to proceed, academicians often must seek out industry collaborators. Industry sponsors must determine whether the area of research aligns with their priorities and whether the benefits of developing a new therapy in a specific disease area outweighs the costs and risks. Occasionally, a biotech firm may spinout from a university and the investigator who owns the intellectual property. These companies often support nascent projects through angel funding, which is start-up funding provided by an individual or a group of individual investors in return for equity in the company. The hope is to develop a candidate drug to the point that the company becomes an attractive investment for venture capitalists. Occasionally, these small biotech firms become cash strapped as drug development proceeds and angel funding begins to dwindle before the company becomes an attractive investment option for venture capitalists. Intellectual property (i.e., the drug in development) is often sold to a larger, more financially viable company; otherwise, development may stop. The Cystic Fibrosis Foundation has tried to bridge the gap between angel funding and venture capitalism through a process known as venture philanthropy. In this process, the CF Foundation supports drug development in return for equity in the sponsor/drug. The CF Foundation has in turn reinvested equity in development programs for other candidate drugs.

There are other mechanisms to encourage industry sponsors to work in $\mathrm{CF}$, including financial benefits conferred by the Orphan Drug Act of 1983. The Orphan Drug Act recognizes that small populations of patients make it difficult for companies to profit from developing drugs for those diseases. The Orphan Drug Act provides benefits that include tax incentives, enhanced patent protections and marketing rights for seven years after approval, clinical research subsidies in the form of grants, FDA support for protocol 
development and study design, and waiving of Prescription Drug User Act filing fees. In addition, many drugs can be granted fast track status from the FDA if they are developed for rare pediatric diseases like CF. Fast track status will reduce the costs for sponsors and shorten the time to approval, thus providing financial incentives for sponsors to develop drugs for CF. These initiatives have induced industry sponsors into CF drug development.

\section{Clinical Development}

Once pre-clinical development has reached a promising stage, the clinical stage of development can follow. All clinical studies must follow standards developed by the International Conference on Harmonisation of Good Clinical Practice (GCP). GCP standards guide the overall conduct of clinical trials, including the reporting of results that are credible and accurate, and ensure that all rights of study subjects are protected.

Clinical trials are generally categorized into four phases. Phase I trials represent the initial in-human studies during drug development. Achieving this step does not guarantee that the drug will be approved(2). The primary aim of Phase I studies is to determine tolerability, pharmacokinetics, and pharmacodynamics in humans to find the appropriate dose range for safety and toxicity. Initial dosing and dose ranges are based on pre-clinical animal studies; the results of Phase I studies identify the dose range that is anticipated to be safe in future clinical studies. Depending on the molecule, pre-clinical results, and mode of delivery (e.g., oral or inhaled), absorption, distribution, metabolism, and/or excretion (ADME) are evaluated. In orphan diseases like CF, some information on efficacy may be sought as a secondary endpoint in Phase I studies. Obtaining some efficacy data may be beneficial to pharmaceutical companies if the data can inform a "go-no-go" decision prior to proceeding to increasingly costly Phase II and III studies. Phase I studies may cost up to $\$ 5$ million, Phase II and Phase III costs may range between $\$ 10$ and $\$ 16$ million and $\$ 10$ and $\$ 30$ million, respectively (https://aspe.hhs.gov/system/files/pdf/77166/rpt_erg.pdf, accessed June 11, 2020).

Phase I studies are most often conducted in a small number, e.g., 20-30, healthy adult volunteers, who are typically male. These studies are very short (a participant may receive the candidate drug for days to weeks), open label, uncontrolled, and are usually conducted at centers that specialize in Phase I trials. Some studies may be conducted in people with the target disease if the mechanism of action is specific to the condition, e.g., chronic endobronchial infection with Pseudomonas aeruginosa in people with $\mathrm{CF}(16)$. Up to $75 \%$ of drug candidates move on to Phase II trials(17).

\section{Safety}

Safety is a focus of all clinical trials, but a top priority in Phase I and early Phase II trials. In the US, the FDA's regulations for IND details safety reporting in clinical trials and the definitions used (https://www.fda.gov/drugs/types-applications/investigational-new-drug-ind-application, accessed June 15, 2020). Adverse Events (AE) are any untoward medical occurrence associated with the use of a drug in humans, whether or not if it is considered to be drug related [IND Application 21 CFR § 312.32 (2020)]. This can be any unfavorable and unintended sign, symptom, laboratory result, or disease that coincides with the use of a drug; there is no implication of causality. AEs are considered Serious AEs (SAE) when the outcome of the AE is death, life-threatening, hospitalization or prolongation of an existing hospitalization, a persistent or significant disability or incapacity, or a congenital abnormality or birth defect. SAEs are reported to the FDA within 7-15 calendar days. In CF clinical trials, hospitalizations are handled as SAEs, even though they occur frequently in people with $\mathrm{CF}$ who are not enrolled in clinical trials and changes in the rates or risk of hospitalizations may be used as an efficacy outcome measure in CF clinical trials. Because $\mathrm{CF}$ is a multiorgan disease with substantial comorbidities, AEs occur commonly in people with CF enrolled in extended duration clinical studies, e.g., $94 \%$ of subjects with $\mathrm{CF}$ in a 96 -week interventional clinical trial had at least one AE;(18) contrasted with a 60 -week study of severe asthma were about $45 \%$ of subjects had at least one $\mathrm{AE}(19)$.

AEs and SAEs are recorded without regards to the potential for causality. All need to be reported during the course of the study. However, the focus of reporting during clinical trials is on events that may not be anticipated, unless mounting evidence suggests causality. An Unexpected Adverse Drug Experience is any 
adverse drug experience that is not included in the prior pre-clinical and clinical trial experience. These may include adverse reactions that are more severe than have been reported previously, e.g., the development of hepatic necrosis would be considered unexpected if prior experience had only demonstrated mild elevation of hepatic enzymes. Suspected Adverse Reactions (SAR) include events where there is a reasonable possibility that the experience may have been caused by the drug. Suspected Unexpected Serious Adverse Reactions (SUSAR) are those events that may be related to the study drug and have not been previously recorded.

Safety monitoring is conducted through a combination of several mechanisms. Institutional review boards (IRB) or Ethics Committees are responsible for evaluating a trial to determine if risks to study participants are appropriate in comparison to the potential anticipated benefits (21 CFR 56.111(a)). Evaluation of the informed consent process is an important component of the responsibilities of the IRB. A medical monitor may work on behalf of the pharmaceutical company to closely monitor study participants. In this role, they evaluate and categorize potential AEs and ensure timely reporting to the sponsor and FDA. A data monitoring committee (DMC) is comprised of a group of individuals with relevant expertise that reviews accumulating data from ongoing clinical trials on a regular basis. The DMC advises the pharmaceutical company regarding the safety of trial participants who remain in the study, as well as future participants. The DMC may recommend that a study stop early for clear signal of benefit or risk.

In clinical trials where investigators are blinded to the treatment assignment (i.e., drug or placebo), unblinding may occur if knowledge of the treatment received is necessary for treating the subject medically or if it will provide critical safety information that could have implications for the ongoing conduct of the trial.

\section{Phase II}

Whereas Phase I studies are typically performed in healthy volunteers, Phase II studies are often the first clinical trials in people with the disease. These studies typically focus on safety, but often include exploratory efficacy measures that are based upon a candidate drug's mechanism of action. In addition to evaluating safety and assessing some efficacy endpoints, other goals of Phase II studies include determining optimal dose and dosing regimens and identification of contraindications. There are no regulatory requirements for conducting Phase II studies. However, they serve an important function by confirming proof-of-concept studies, helping to reduce risks to participants prior to larger Phase III studies, and can streamline future Phase III studies, e.g., by selecting a single dose level.

Initial Phase II studies are typically in adults. It is important to start with adults as they understand the risks associated with participation in a research study. Once enough safety data are obtained in adults, studies then proceed to children. Children of all ages may be enrolled simultaneously, but it is often customary to enroll children ages 12 to 17 years first, then 6-11 years, followed by 2-5 years, and finally under two years of age, if it is anticipated that a candidate drug will benefit patients in the youngest age groups. Phase II trials are often complex and may incorporate specialized outcome measures. These trials frequently are conducted at research centers that are expert in Phase II studies.

Phase II studies are often double-blind, and there are usually two general treatment groups: drug-treated vs. placebo-treated. While Phase II studies are primarily safety studies, they often include a dose ranging design in order to choose the best doses to be used in the next study. Phase II studies may be single ascending dose (SAD) or multiple ascending dose (MAD). In a SAD trial, subjects are randomized to placebo or a drug cohort. The first drug cohort gets 1 dose of the lowest dose of the drug or placebo. A review of the safety data for the first dose cohort by the data safety monitoring board (DSMB) will occur, and if the study is determined to be safe, enrollment in the next highest dose cohort will begin. This process will continue as long as each proceeding dose cohort is safe or until the study ends. A MAD study is similar to a SAD study, but instead of getting just one dose of the candidate drug, study participants will receive multiple doses of the same dose of drug or placebo over time before the next highest dose cohort is enrolled. The number of study subjects in a Phase II clinical trial is variable. A trial that employees a SAD design may have less than 20 subjects whereas a MAD design may enroll over 400 subjects. As the primary objective for Phase II studies is safety and tolerability, the outcome measures reflect that. Typical Phase II safety outcomes include 
but are not limited to subject diaries, subject quality of life questionnaires, adverse events, vital signs, safety laboratories (electrolytes, liver function tests, renal function tests, CBC with differential count, urinalysis), ECG, radiographic imaging, pulmonary function testing, and occurrence of pulmonary exacerbations. While many people consider measures of lung function, changes in quality of life questionnaires, and frequency or time to pulmonary exacerbations efficacy outcomes, in Phase II studies, they are often considered safety outcomes to ensure that the investigational product under evaluation does not cause an acute worsening of underlying disease. Most Phase II studies are of insufficient duration or power to evaluate these parameters as measures of efficacy. Lack of a positive efficacy signal in Phase II often does not stop the development of a candidate drug. However, demonstration of negative effects and safety concerns will stop the development. Occasionally investigators will sub-classify Phase II studies into Phase IIa and Phase IIb. Phase IIa studies are often shorter (4-8 weeks) smaller pilot studies that are dose ranging in nature and focus almost exclusively on safety and tolerability with some exploratory efficacy measures. Phase IIb studies are larger, often longer (12 to 24 weeks) studies that focus on both safety and efficacy. These studies are frequently used by study sponsors to support the efficacy of their candidate drug in seeking regulatory approval, and usually significantly more expensive. The results of Phase IIb studies are critical in determining whether a candidate drug should go on to a much larger, longer, and more expensive Phase III registration study. Therefore, the sites that conduct Phase II studies must be experts in clinical trials and produce data of the highest quality.

Proof-of-concept (POC) or proof-of principle studies bridge Phase I and Phase II dose ranging/safety studies. POC studies are typically small, short, biomarker-based studies. POC studies are designed to detect a signal in a disease-relevant mechanism or biomarker relevant to the disease. POC studies assess changes in biologic outcomes which would presumably translate into clinical benefit. A biomarker is defined as " $a$ characteristic that is objectively measured and evaluated as an indicator of normal biologic processes, pathogenic processes, or pharmacologic response to a therapeutic intervention" (20). With respect to efficacy biomarkers for both Phase II studies and POC studies, selection depends upon a candidate drug's mechanism of action. A biomarker in a clinical trial of CFTR modulator likely would not be useful in a clinical trial of an antiinflammatory drug. Biomarkers that have been used in the past based upon where in the pathophysiologic cascade a drug exerts its affect are given in the Table. POC studies provide preliminary data on clinically relevant endpoints. The stronger the link between the biomarker and a clinical endpoint, the more likely that biomarker can be used in later phase studies(21). Whereas the typical Phase II study includes a placebo group and a drug treatment group, this is not always the case with POC studies in which there might not be a placebo group. In the absence of a placebo group, POC studies are usually open label; end-of-treatment outcomes are compared to their pre-treatment values in this design. This reduces the number of subjects that need to be enrolled in the study as well as the duration of the study, thereby reducing the overall cost. These trials frequently are too small to provide significant data about the clinical efficacy of a candidate drug, and in fact, POC studies may not reach statistical significance for the targeted biomarkers. The question then becomes whether trends in the right direction for a panel of biomarkers provides enough information to make a decision to proceed to Phase II safety studies, particularly if the POC study is being used as a method to screen for the most promising candidates to move forward. It is entirely possible that a beneficial therapy might be discarded if it doesn't show any trends in a small, quick POC study.

One example that illustrates the risk of POC studies is the low-hanging fruit project initiated in the early millennium. In this project, anti-inflammatory drugs methotrexate, hydroxychloroquine, pioglitazone, and simvastatin, which were approved for other non-CF pro-inflammatory diseases were evaluated in people with CF. In these studies, subjects provided induced sputum samples at the beginning and end of 1-month of treatment to determine if the drug impacted inflammatory markers in CF sputum. None of the drugs demonstrated statistically significant differences in any inflammatory marker when post-treatment values were compared to pre-treatment values. High-dose ibuprofen was also studied using a similar study design because it was the only currently recommended anti-inflammatory drug for $\mathrm{CF}(22)$. Although it was already used clinically to treat inflammation in $\mathrm{CF}$, high-dose ibuprofen demonstrated only trends in changes in sputum neutrophils and a statistically significant decrease in sputum IL-6(23). One wonders - had antiinflammatory drugs been screened by changes of inflammatory markers in induced sputum in the 1980s, 
would ibuprofen have moved forward to a clinical trial? This then leads to the concern that other potentially beneficial anti-inflammatory drugs may have been removed from consideration using this study design. It is likely that the study design for the low hanging fruit project was flawed for a variety of reasons: other than ibuprofen, the anti-inflammatory dose of each drug for CF was unknown, the study was underpowered to detect a difference in stable subjects, the study duration was too brief to detect a drug's anti-inflammatory effect, and sputum measures were too variable. However, if the studies were larger and longer, the benefit of using sputum as a screening tool diminishes.

\section{Phase III}

Phase III trials are the pivotal and confirmatory clinical trials required to demonstrate benefit and support approval of a new therapy. While safety continues to be carefully monitored, the primary focus of Phase III trials is efficacy. These studies are conducted in a randomized, blinded fashion and compare the candidate drug either to a placebo or, if it would not be considered ethical to withhold, standard treatment. The scope and design of Phase III trials are often negotiated with regulators prior to trial initiation. The candidate drug must be manufactured and administered in the same manner as would be expected after completion of the clinical trial. In CF, these studies may enroll anywhere from 200 to 600 participants, though these studies may be much larger in more common disease conditions. Phase III trials require multiple institutions to participate, and may include international sites. Two successful "adequate and well-controlled" (21 CFR 314.126) Phase III clinical trials are typically required to obtain FDA approval; these may be conducted in parallel. In some cases, regulatory agencies have negotiated with sponsors to consider drug approval with a single Phase III trial if there is a supporting Phase II study conducted under similar conditions, i.e., randomized, controlled, blinded, etc. Because $\mathrm{CF}$ drugs have been chronic therapies, extended safety data is needed, even when clinical benefit is seen after a short interval (e.g., CFTR modulators). This has led to most studies being at least 6 months long. In addition, GCP requires 12 months of drug exposure to assess safety, so many studies have open-label follow up evaluations.

\section{Clinical Trial Endpoints}

To gain regulatory approval, Phase III trials need to evaluate accepted clinical endpoints, which directly measures how a patient feels, functions, or survives. Survival is not a pragmatically feasible endpoint in the development of chronic CF drugs because these trials would take years and only enroll patients with the most severe lung disease. A surrogate endpoint is a laboratory measure or a physical sign that can substitute for a clinically meaningful endpoint(24). Surrogate endpoints may be used if they are strongly associated with direct clinical endpoints and have been validated. Lung function is a surrogate endpoint accepted by regulatory agencies based on the relationship between lung function and mortality $(25)$. However, how changes in lung function are measured will influence study design. Most CF drugs have been approved on the basis of an acute improvement in forced expiratory volume in 1 second $\left(\mathrm{FEV}_{1}\right)$, evaluated after weeks-months. While an acute improvement in $\mathrm{FEV}_{1}$ may be beneficial to people with $\mathrm{CF}$, a more meaningful goal of chronic $\mathrm{CF}$ therapies would be to slow the decline in lung function. This has been shown for a few CF therapies, $(26$, 27 ) but these studies can take years to detect a meaningful slowing of lung function decline. Longer studies may require fewer patients, but such studies are also difficult to manage and can become very costly. In the era of highly effective modulator therapy (HEMT), the number of patients required in any study in CF may increase significantly as health outcomes improve $(28,29)$.

Deciding between evaluating acute improvements in $\mathrm{FEV}_{1}$ or slowing the chronic decline of lung function may depend on both the intervention being studied and the patient population of interest. Patients with mild disease may not be able to demonstrate acute improvements in $\mathrm{FEV}_{1}$ (the so-called ceiling effect); these patients may also experience the fastest decline in lung function(30). Thus, drugs designed to address early lung disease or prevent the development of more severe disease, such as anti-inflammatory therapies, may opt for longer studies in healthier patients. More recent studies have focused on acute improvements in $\mathrm{FEV}_{1}$, and then been followed by prolonged open-label, observational studies (see more details in Phase $I V$ below), but as overall lung function improves, demonstrating acute improvements in $\mathrm{FEV}_{1}$ may become more difficult(29). 
Reduction in the risk or rate of pulmonary exacerbations would seem to be an attractive clinical endpoint to demonstrate efficacy of a new drug because they directly affect how a patient feels, functions, and in people with severe lung disease, survives $(31,32)$. However, pulmonary exacerbations have proven problematic for several reasons(33). First, there is no widely accepted and validated prospective definition. Several definitions have been put forward and used in clinical studies $(6,34,35)$; the FDA has seemed to prefer a version of the Fuchs criteria first used in the pivotal study of dornase alfa. Second, there are several methods for determining changes in pulmonary exacerbations: time to the first pulmonary exacerbation, frequency of pulmonary exacerbation, total number of pulmonary exacerbations, etc. Time to the first pulmonary exacerbation requires the smallest number of participants, but may not be considered an acceptable outcome for registration studies by regulatory agencies. Alternative measures of pulmonary exacerbation require larger numbers of patients; this can be mitigated by enriching a study population for patients who have a higher risk of pulmonary exacerbations, but these patients may not be the ideal population for the drug, and such a study could lack generalizability to the wider CF population.

Patient reported outcomes (PROs) that directly measures a patient's health or their quality of life without interpretation by medical professionals may be validated as surrogate endpoints(36). There are several CFrelated PRO tools (e.g., CFQ-R, CFRSD) $(37,38)$, and improvement in symptoms using the CFQ-R was the

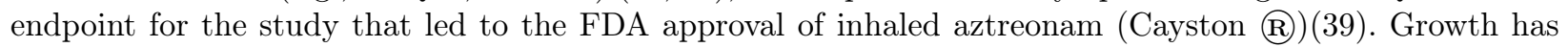
been used in the past as a validated surrogate endpoint, though not in any recent drug trials(40). As CFTR modulator studies expand to younger population, treatment associated effects on linear growth is affected may become of interest(41).

The use of surrogate endpoints such as PROs may present difficulties with study interpretation(24). Outside of CF, pivotal studies may be so large that even small clinical differences may be statistically significant. The difference may be less important in pediatric and/or orphan disease studies, because these are typically smaller studies and any statistically significant results are likely to also be clinically meaningful. However, determining what difference is clinically meaningful is important in many situations. An example would be a non-inferiority study, which is designed to show that a new therapy is not unacceptably worse than current standard therapy. This situation may arise in CF when comparing a new drug within the same therapeutic class (e.g., CFTR modulator or new inhaled antibiotic) to an established drug when comparison with a placebo would not be acceptable(42). Clinician surveys have indicated that many are uncomfortable withdrawing efficacious medications(43).

Biomarkers may be used as surrogate endpoints and could potentially speed up drug development. Biomarkers require validation to show they reflect the biologic activity of a therapy as well as the relationship with clinical outcomes(21). Biomarkers that have been explored in CF include those that would reflect changes in CFTR activity (e.g., sweat chloride, nasal potential difference, intestinal current measurement), infection (e.g., bacterial density, detection of CF pathogens, and inflammation (e.g., sputum neutrophil elastase activity, cell counts, cytokines, and serum CRP). In order to validate biomarkers as useful clinical surrogate endpoints, the relationships between biomarker changes/clinically meaningful changes or thresholds and the likelihood of subsequent clinical benefit must be clearly understood(24). Otherwise, the use of biomarkers is limited because risks and benefits cannot be fully assessed.

Recently, the FDA has demonstrated willingness to expand the use of results from biomarker studies in the approval process for CFTR modulators. Theratyping, the process of matching medications to specific CFTR mutations based upon in vitro testing results, has been used to expand the indication for ivacaftor beyond the original mutations for which it was approved through the traditional regulatory approval pathway(44). In 2017, after ivacaftor had been approved by the FDA for people with at least one G551D mutation and deemed safe in people with $\mathrm{CF}$, ivacaftor was approved for people with $\mathrm{CF}$ with one of 23 other residual function mutations based on how cells from people with CF responded to ivacaftor in laboratory experiments(45). Similarly, elexacaftor/tezacaftor/ivacaftor has been approved for all CFTR mutations that can be demonstrated to respond in laboratory experiments and has not been restricted to just those mutations present in clinical trial participants. 
Identifying appropriate clinical trial endpoints in young children may be particularly difficult. Many endpoints that may be used in studies in adults occur less frequently (e.g., deaths, pulmonary exacerbations), have not been validated (e.g., age-appropriate PROs), or cannot be measured readily (e.g., lung function in young children). Pivotal Phase III studies have been pursued in older adolescents and adults in part to avoid these limitations. Once drugs have been approved for use in older children, the FDA has relied on safety data alone as the primary endpoint in clinical trials in younger children with $\mathrm{CF}(46-48)$. This has the added benefit of significantly reducing study size: pivotal trials of ivacaftor included 100 patients 12 years and above, but only 38 children ages $2-5$ years $(48,49)$. However, this strategy may not allow for the detection of rare adverse events.

\section{FDA approval}

After successful completion of the required pivotal Phase III studies that demonstrate efficacy, drug developers submit a New Drug Application (NDA) to the FDA. Standard review is approximately 12 months, though more rapid reviews are becoming more common through specific pathways developed by the FDA (https://www.fda.gov/patients/learn-about-drug-and-device-approvals/fast-trackbreakthrough-therapy-accelerated-approval-priority-review, accessed June 15, 2020). These are reserved for priority drugs that represent an advance in treatment, diagnosis, or disease prevention when compared to

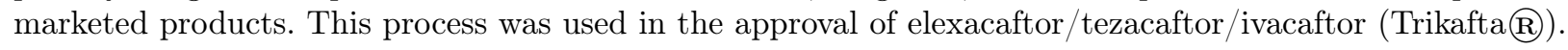

\section{Phase IV}

Phase IV clinical trials, also known as post-marketing surveillance trials, collect real-world data and occur after FDA drug approval. A Phase IV trial is typically part of a safety surveillance program (pharmacovigilance) once a drug is on the market. A Phase IV trial typically has the fewest risks for participants because the drug has been studied in several other earlier phase clinical trials. Typically, participants do not have to enroll in a Phase IV clinical trial, and they receive all of the usual disease treatments. Another advantage of a Phase IV trial is that it collects data from many different patient populations who receive care at dozens of different sites, thus increasing the robustness of the drug's overall clinical trial data. A Phase IV study may be required by the FDA to fill in knowledge gaps, or it may be initiated by the sponsor to gather more information about the drug and how its use might be expanded. The goal of a Phase IV trial is to collect long-term safety and efficacy data in large groups of people and to identify rare adverse events that may not have been seen in smaller earlier phase studies. In addition, a sponsor may initiate a Phase IV trial to collect data to expand the label of the drug to different groups with the same disease or for new indications in other diseases with a similar pathophysiology, to obtain quality of life data, to evaluate drug-drug interactions, to compare the drug to other drugs on the market, and to analyze the cost-effectiveness of the drug. Because one of the goals is to collect information on rare adverse events, these trials often capture data on large numbers of study participants for long periods of time (2 to 5 years or more). For example, the Phase IV data from lumacaftor/ivacaftor demonstrated that $17 \%$ of people discontinued the drug within 6 months of starting it compared to only $4 \%$ of participants in the randomized control trials, and $39 \%$ of people with severe lung disease (FEV1 percent predicted $<40 \%$ ) demonstrated higher rates of serious adverse events (39\% vs. $17 \%$ in RCTs) and had higher rates of discontinuation of the drug(50). These examples highlight the importance of why sponsors must ensure a Phase IV trial is not under-powered. In addition, significant adverse events may be identified during a Phase IV trial that result in the drug being removed from the market.

\section{Conclusion}

Since the approval of dornase alfa in 1993, several drugs have been developed and approved by regulatory agencies specifically for people with CF. This process has (so far) culminated in 2020, with approval of highlyeffective modulators for approximately $90 \%$ of people with CF ages 12 years and older. These transformative therapies will surely reduce morbidity and extend longevity, yet not all people with CF will have access to these drugs. The therapeutic development pipeline contains candidate drugs that address many different aspects of disease, although many face particular challenges to being approved. However, the challenges of 
drug development must be met until that time when a one-time cure is available to all people with CF.

\section{Acknowledgements}

The authors wish to acknowledge Donald R. VanDevanter, Ph.D. for reviewing and commenting on the manuscript.

Table. Examples of Biomarkers Used in Phase II CF Clinical Trials Based upon Pathophysiology

\begin{tabular}{|c|c|}
\hline Respiratory System & Biomarker \\
\hline Abnormal gene & $\begin{array}{l}\text { Gene transfection in nasal or bronchial epithelial } \\
\text { cells Nasal potential difference }\end{array}$ \\
\hline Abnormal protein & $\begin{array}{l}\text { Sweat Chloride Nasal potential difference Intestinal } \\
\text { current measurement }\end{array}$ \\
\hline Abnormal airway environment & Mucociliary and cough clearance \\
\hline Mucus obstruction & Mucociliary and cough clearance Mucus rheology \\
\hline Infection & Microbiology Microbiome \\
\hline Inflammation & $\begin{array}{l}\text { Inflammatory mediators in sputum or } \\
\text { bronchoalveolar lavage fluid Inflammatory } \\
\text { mediators in serum Measures in exhaled breath }\end{array}$ \\
\hline $\begin{array}{l}\text { Damage to airway wall architecture } \\
\text { Gastrointestinal System }\end{array}$ & Radiographic imaging Structural elements in urine \\
\hline Pancreatic insufficiency & $\begin{array}{l}\text { Coefficient of fat absorption Coefficient of Nitrogen } \\
\text { absorption Circulating concentrations of vitamins }\end{array}$ \\
\hline
\end{tabular}

Figure 1. Pathogenesis of CF lung disease. This schematic provides a thought construct of how the abnormal gene leads to lung destruction by passing through the vicious cycle of airway obstruction, chronic bacterial infection, and persistent inflammation. Therapies are being developed to address the different levels of pathogenesis. ASF = airway surface fluid.

\section{Hosted file}

image1.emf available at https://authorea.com/users/337201/articles/465214-drug-developmentfor-cystic-fibrosis

Figure 2. Drug development overview. A summary of stages in the development of a candidate drug, from pre-clinical to post-marketing evaluations. Regulatory applications are given in the pink boxes. IND = investigational new drug; NDA = new drug application.

\section{Hosted file}

image2.emf available at https://authorea.com/users/337201/articles/465214-drug-developmentfor-cystic-fibrosis

\section{References}

1. Kraljevic S, Stambrook PJ, Pavelic K. Accelerating drug discovery. EMBO Rep. 2004;5(9):837-42.

2. Hay M, Thomas DW, Craighead JL, Economides C, Rosenthal J. Clinical development success rates for investigational drugs. Nat Biotechnol. 2014;32(1):40-51.

3. DiMasi JA, Grabowski HG, Hansen RW. Innovation in the pharmaceutical industry: New estimates of R\&D costs. J Health Econ. 2016;47:20-33.

4. MedicineNet. Drug Approvals - From Invention to Market ... A 12-Year Trip 1999, July 14 [Available from: https://www.medicinenet.com/script/main/art.asp?articlekey $=9877$. 
5. Goss CH, Mayer-Hamblett N, Kronmal RA, Ramsey BW. The cystic fibrosis therapeutics development network (CF TDN): a paradigm of a clinical trials network for genetic and orphan diseases. Adv Drug Deliv Rev. 2002;54(11):1505-28.

6. Fuchs H, Borowitz D, Christiansen D, Morris E, Nash M, Ramsey B, et al. Effect of aerosolized recombinant human DNase on exacerbations of respiratory symptoms and on pulmonary function in patients with cystic fibrosis. The Pulmozyme Study Group. N Engl J Med. 1994;331(10):637-42.

7. Middleton PG, Mall MA, Drevinek P, Lands LC, McKone EF, Polineni D, et al. Elexacaftor-TezacaftorIvacaftor for Cystic Fibrosis with a Single Phe508del Allele. N Engl J Med. 2019;381(19):1809-19.

8. Chmiel JF, Konstan MW. Anti-inflammatory medications for cystic fibrosis lung disease: selecting the most appropriate agent. Treat Respir Med. 2005;4(4):255-73.

9. Chmiel JF, Konstan MW. Inflammation and anti-inflammatory therapies for cystic fibrosis. Clin Chest Med. 2007;28(2):331-46.

10. Chmiel JF, Konstan MW, Elborn JS. Antibiotic and anti-inflammatory therapies for cystic fibrosis. Cold Spring Harb Perspect Med. 2013;3(10):a009779.

11. Konstan MW, Doring G, Heltshe SL, Lands LC, Hilliard KA, Koker P, et al. A randomized double blind, placebo controlled phase 2 trial of BIIL 284 BS (an LTB4 receptor antagonist) for the treatment of lung disease in children and adults with cystic fibrosis. J Cyst Fibros. 2014;13(2):148-55.

12. Doring G, Bragonzi A, Paroni M, Akturk FF, Cigana C, Schmidt A, et al. BIIL 284 reduces neutrophil numbers but increases $\mathrm{P}$. aeruginosa bacteremia and inflammation in mouse lungs. J Cyst Fibros. 2014;13(2):156-63.

13. Torphy TJ, Allen J, Cantin AM, Konstan MW, Accurso FJ, Joseloff E, et al. Considerations for the Conduct of Clinical Trials with Antiinflammatory Agents in Cystic Fibrosis. A Cystic Fibrosis Foundation Workshop Report. Ann Am Thorac Soc. 2015;12(9):1398-406.

14. National Institutes of Health. Definitions 2015, April 7 [Available from: https://www.fda.gov/inspections-compliance-enforcement-and-criminal-investigations/fda-bioresearchmonitoring-information/definitions.

15. Cook N, Hansen AR, Siu LL, Abdul Razak AR. Early phase clinical trials to identify optimal dosing and safety. Mol Oncol. 2015;9(5):997-1007.

16. Goss CH, Kaneko Y, Khuu L, Anderson GD, Ravishankar S, Aitken ML, et al. Gallium disrupts bacterial iron metabolism and has therapeutic effects in mice and humans with lung infections. Sci Transl Med. 2018;10(460).

17. Takebe T, Imai R, Ono S. The Current Status of Drug Discovery and Development as Originated in United States Academia: The Influence of Industrial and Academic Collaboration on Drug Discovery and Development. Clin Transl Sci. 2018;11(6):597-606.

18. McKone EF, Borowitz D, Drevinek P, Griese M, Konstan MW, Wainwright C, et al. Long-term safety and efficacy of ivacaftor in patients with cystic fibrosis who have the Gly551Asp-CFTR mutation: a phase 3, open-label extension study (PERSIST). The Lancet Respiratory Medicine. 2014;2(11):902-10.

19. Busse WW, Morgan WJ, Gergen PJ, Mitchell HE, Gern JE, Liu AH, et al. Randomized trial of omalizumab (anti-IgE) for asthma in inner-city children. N Engl J Med. 2011;364(11):1005-15.

20. NIH Definitions Working Group. Biomarkers and surrogate endpoints in clinical research: definitions and conceptual model. . In: Downing G, editor. Biomarkers and Surrogate Endpoints: Clinical Research and Applications Amsterdam: Elsevier; 2000. p. 1-9. 
21. Muhlebach MS, Clancy JP, Heltshe SL, Ziady A, Kelley T, Accurso F, et al. Biomarkers for cystic fibrosis drug development. J Cyst Fibros. 2016;15(6):714-23.

22. Mogayzel PJ, Jr., Naureckas ET, Robinson KA, Mueller G, Hadjiliadis D, Hoag JB, et al. Cystic fibrosis pulmonary guidelines. Chronic medications for maintenance of lung health. Am J Respir Crit Care Med. 2013;187(7):680-9.

23. Chmiel JF, Konstan MW, Accurso FJ, Lymp J, Mayer-Hamblett N, VanDevanter DR, et al. Use of ibuprofen to assess inflammatory biomarkers in induced sputum: Implications for clinical trials in cystic fibrosis. J Cyst Fibros. 2015;14(6):720-6.

24. Fleming TR, Powers JH. Biomarkers and surrogate endpoints in clinical trials. Statistics in medicine. 2012;31(25):2973-84.

25. Mayer-Hamblett N, Rosenfeld M, Emerson J, Goss C, Aitken M. Developing cystic fibrosis lung transplant referral criteria using predictors of 2-year mortality. Am J Respir Crit Care Med. 2002;166(12 Pt 1):1550-5.

26. Konstan MW, Byard PJ, Hoppel CL, Davis PB. Effect of high-dose ibuprofen in patients with cystic fibrosis. N Engl J Med. 1995;332(13):848-54.

27. Sawicki GS, McKone EF, Pasta DJ, Millar SJ, Wagener JS, Johnson CA, et al. Sustained Benefit from ivacaftor demonstrated by combining clinical trial and cystic fibrosis patient registry data. Am J Respir Crit Care Med. 2015;192(7):836-42.

28. VanDevanter DR, Konstan MW. Outcome measures for clinical trials assessing treatment of cystic fibrosis lung disease. Clin Investig (Lond). 2012;2(2):163-75.

29. Stanojevic S, Ratjen F. Physiologic endpoints for clinical studies for cystic fibrosis. J Cyst Fibros. 2016;15(4):416-23.

30. Konstan M, Morgan W, Butler S, Pasta D, Craib M, Silva S, et al. Risk factors for rate of decline in forced expiratory volume in one second in children and adolescents with cystic fibrosis. J Pediatr. 2007;151(2):134-9, 9.e1.

31. Ellaffi M, Vinsonneau C, Coste J, Hubert D, Burgel P, Dhainaut J, et al. One-year outcome after severe pulmonary exacerbation in adults with cystic fibrosis. Am J Respir Crit Care Med. 2005;171(2):158-64.

32. Britto M, Kotagal U, Hornung R, Atherton H, Tsevat J, Wilmott R. Impact of recent pulmonary exacerbations on quality of life in patients with cystic fibrosis. Chest. 2002;121(1):64-72.

33. Vandevanter DR, Yegin A, Morgan WJ, Millar SJ, Pasta DJ, Konstan MW. Design and powering of cystic fibrosis clinical trials using pulmonary exacerbation as an efficacy endpoint. J Cyst Fibros. 2011;10(6):453-9.

34. Goss C, Burns J. Exacerbations in cystic fibrosis. 1: Epidemiology and pathogenesis. Thorax. 2007;62(4):360-7.

35. Treggiari MM, Rosenfeld M, Mayer-Hamblett N, Retsch-Bogart G, Gibson RL, Williams J, et al. Early anti-pseudomonal acquisition in young patients with cystic fibrosis: rationale and design of the EPIC clinical trial and observational study'. Contemp Clin Trials. 2009;30(3):256-68.

36. Guidance for industry: patient-reported outcome measures: use in medical product development to support labeling claims: draft guidance. Health Qual Life Outcomes. 2006;4:79.

37. Quittner AL, Buu A, Messer MA, Modi AC, Watrous M. Development and Validation of the Cystic Fibrosis Questionnaire in the United States: A Health-Related Quality-of-Life Measure for Cystic Fibrosis. Chest. 2005;128(4):2347-54.

38. Goss CH, Caldwell E, Gries KC, Leidy NK, Edwards T, Flume PA, et al. Validation of a novel patient-reported respiratory symptoms instrument in cystic fibrosis: CFRSD-CRISS. Pediatric Pulmonology. 2013;48(S36):A251. 
39. Retsch-Bogart G, Quittner A, Gibson R, Oermann C, McCoy K, Montgomery A, et al. Efficacy and safety of inhaled aztreonam lysine for airway pseudomonas in cystic fibrosis. Chest. 2009;135(5):1223-32.

40. Ramsey B, Boat T. Outcome measures for clinical trials in cystic fibrosis. Summary of a Cystic Fibrosis Foundation consensus conference. J Pediatr. 1994;124(2):177-92.

41. Stalvey MS, Pace J, Niknian M, Higgins MN, Tarn V, Davis J, et al. Growth in Prepubertal Children With Cystic Fibrosis Treated With Ivacaftor. Pediatrics. 2017;139(2).

42. VanDevanter DR, Mayer-Hamblett N. Innovating cystic fibrosis clinical trial designs in an era of successful standard of care therapies. Current opinion in pulmonary medicine. 2017;23(6):530-5.

43. VanDevanter DR, Mayer-Hamblett N, Boyle M. Feasibility of placebo-controlled trial designs for new CFTR modulator evaluation. J Cyst Fibros. 2017;16(4):496-8.

44. Clancy JP, Cotton CU, Donaldson SH, Solomon GM, VanDevanter DR, Boyle MP, et al. CFTR modulator theratyping: Current status, gaps and future directions. J Cyst Fibros. 2019;18(1):22-34.

45. Durmowicz AG, Lim R, Rogers H, Rosebraugh CJ, Chowdhury BA. The U.S. Food and Drug Administration's Experience with Ivacaftor in Cystic Fibrosis. Establishing Efficacy Using In Vitro Data in Lieu of a Clinical Trial. Ann Am Thorac Soc. 2018;15(1):1-2.

46. Rosenfeld M, Wainwright CE, Higgins M, Wang LT, McKee C, Campbell D, et al. Ivacaftor treatment of cystic fibrosis in children aged 12 to $<24$ months and with a CFTR gating mutation (ARRIVAL): a phase 3 single-arm study. The Lancet Respiratory medicine. 2018;6(7):545-53.

47. Milla CE, Ratjen F, Marigowda G, Liu F, Waltz D, Rosenfeld M. Lumacaftor/Ivacaftor in Patients Aged 6-11 Years With Cystic Fibrosis Homozygous for F508del-CFTR. Am J Respir Crit Care Med. 2016.

48. Davies JC, Cunningham S, Harris WT, Lapey A, Regelmann WE, Sawicki GS, et al. Safety, pharmacokinetics, and pharmacodynamics of ivacaftor in patients aged 2-5 years with cystic fibrosis and a CFTR gating mutation (KIWI): an open-label, single-arm study. The Lancet Respiratory medicine. 2016;4(2):107-15.

49. Ramsey BW, Davies J, McElvaney NG, Tullis E, Bell SC, Dřevínek P, et al. A CFTR potentiator in patients with cystic fibrosis and the G551D mutation. N Engl J Med. 2011;365(18):1663-72.

50. Burgel PR, Munck A, Durieu I, Chiron R, Mely L, Prevotat A, et al. Real-Life Safety and Effectiveness of Lumacaftor-Ivacaftor in Patients with Cystic Fibrosis. Am J Respir Crit Care Med. 2020;201(2):188-97. 

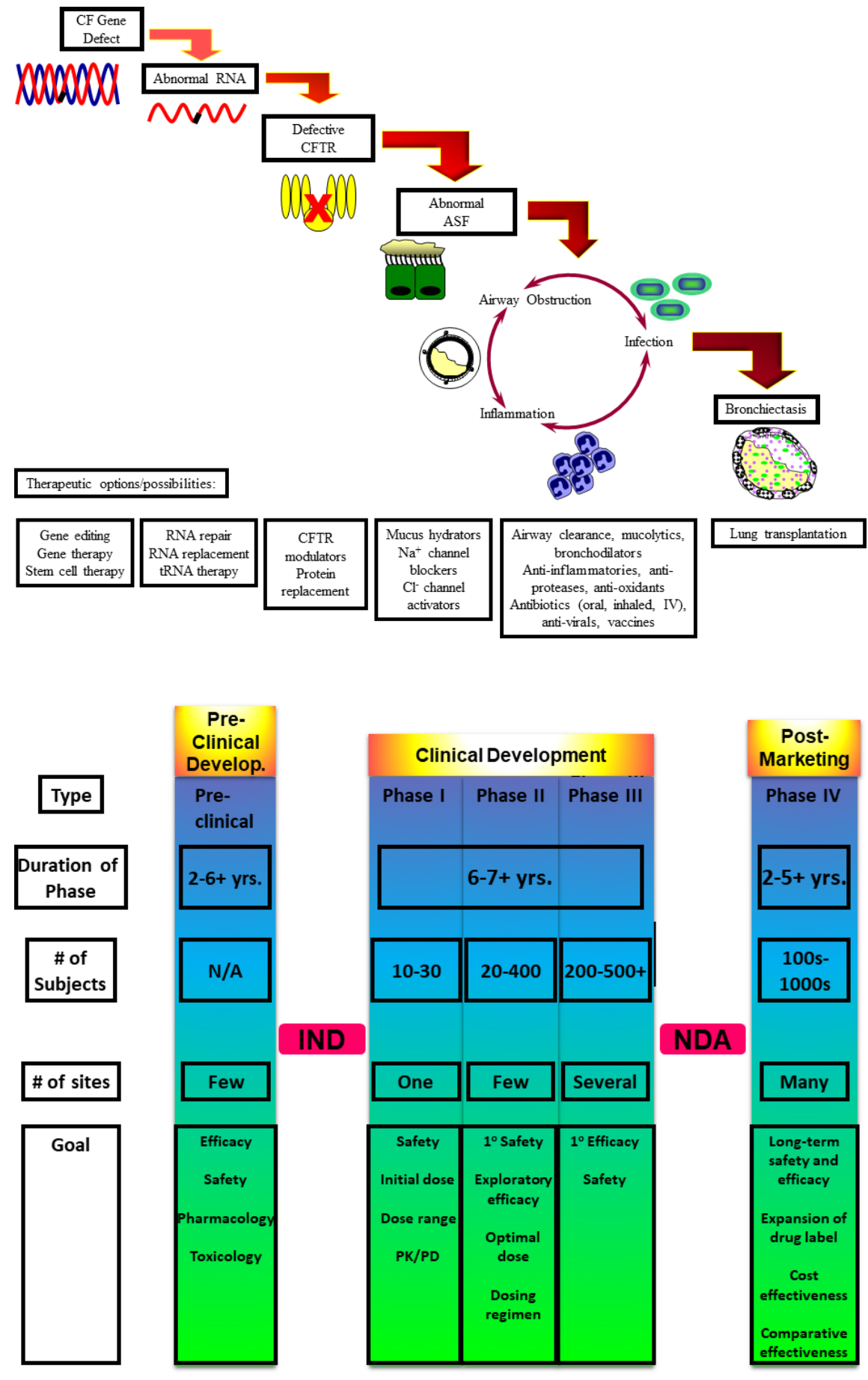

Hosted file

Drug Development Table.docx available at https://authorea.com/users/337201/articles/465214drug-development-for-cystic-fibrosis 